\title{
AUMENTO DA CONFIABILIDADE OPERACIONAL E DISPONIBILIDADE DO FEA DA VOTORANTIM RESENDE ATRAVÉS DO USO DAS TECNOLOGIAS DOUBLE SIDE E PROTECT BLOCK*
}

\section{Resumo}

Thiago Pinto Wandekoken ${ }^{1}$ Pedro Henrique Coutinho ${ }^{2}$ Júlio César Guimarães ${ }^{3}$ Claysson Pereira da Costa ${ }^{4}$ José Fernando Cerqueira ${ }^{5}$

A aciaria elétrica da Votorantim Siderurgia em Resende, possui um FEA de capacidade de $100 \mathrm{t}$, transformador de 100+20 MVa, equipado com 05 injetores convencionais de oxigênio e 03 injetores convencionais de carbono. Em função do elevado aporte de energia química e elétrica inserida no processo os painéis refrigerados, e demais equipamentos localizados em determinadas regiões do FEA, são termicamente mais solicitados e requerem maior atenção e rigor do sistema de manutenção preventiva, a fim de evitar interrupções operacionais do processo. Através da análise de dados históricos, detectou-se que a região do FEA de maior criticidade e intervenções de manutenção era o painel refrigerado $\mathrm{P}-10$. As tecnologias Double Side e Protect Block foram instaladas em substituição ao painel refrigerado P-10 com o objetivo de aumentar a confiabilidade operacional do FEA, reduzir os riscos de acidentes operacionais, aumentar a disponibilidade dos equipamentos nesta posição, reduzir o número de intervenções de manutenção (preventiva e corretiva), reduzir os custos de manutenção (preventiva e corretiva) e consequentemente reduzir o power off do FEA.

Palavras-chave: Double side; Protect block; Aumento de confiabilidade; Redução de custos.

\section{OPERATIONAL RELIABILITY INCREASE AND AVAILABILITY OF VOTORANTIM'S EAF THROUGH THE USE OF DOUBLE SIDE AND PROTECK BLOCK TECHNOLOGIES}

\section{Abstract}

The steel meltshop of Votorantim Resende has an EAF with $100 \mathrm{t}$ capacity, a 100+20 MVa electrical transformer, equipped with 05 conventional oxygen injectors and 03 conventional carbon injectors. Due to the high chemical and electrical energy use in the process the water cooled panels, and others equipments located in the hot spots of the furnace are most thermal stressed and needs more attention and prior maintenance preventive system to avoid the EAF interruptions. The analysis of historical data shown that the most critical water cooled panel of the EAF was a Position 10. In order to increase the operational reliability, reduce the accidents risks, increased the availability, reduce maintenance interventions, reduce the costs and reduce power off time, the panel in the Position 10 was replaced by Double Side and Protect Block technologies.

Keywords: Double Side; Protect Block; Reliability increase; Cost reduction.

1 Eng. Mecatrônico, Mestre em Engenharia Metalúrgica e de Materiais, Engenheiro de Aplicações, Lumar Metals, Ipatinga, MG, Brasil.

Eng. Metalurgista, Engenheiro de Processos Pleno, Votorantim Siderurgia, Resende, RJ, Brasil.

Técnico Mecânico, Técnico Especialista, Votorantim Siderurgia, Resende, RJ, Brasil

Técnico Mecânico, Técnico Especialista, Votorantim Siderurgia, Resende, RJ, Brasil

Técnico Mecânico, Técnico Especialista, Votorantim Siderurgia, Resende, RJ, Brasil. 


\section{INTRODUÇÃO}

Inicialmente, os painéis refrigerados foram instalados nas paredes laterais dos FEA em substituição aos revestimentos refratários, com a finalidade de diminuir o tempo de paralisação dos fornos para reparos. Os excelentes resultados alcançados induziram à ampliação da substituição para toda a parede e até mesmo para as abóbadas, região externa em volta do miolo [1].

De acordo com a orientação de montagem dos tubos dos painéis refrigerados eles podem ser classificados como: Painéis de Tubos Horizontais, Painéis de Tubos Verticais e Painéis de Tubos Mistos. Os painéis refrigerados também são classificados em relação à quantidade de painéis que um mesmo painel refrigerado possui, sendo classificados como Painéis Simples e Painéis Duplos.

Os painéis duplos possuem duas fileiras de tubos em sua construção, constituindo dois painéis distintos / independentes fixados em um mesmo painel refrigerado. $O$ painel que fica mais exposto ao interior do forno é chamado de "Painel Frontal", ou "Painel Interno" e o painel que fica mais próximo à carcaça do forno é chamado de "Painel Traseiro", ou "Painel Externo".

Em meio ao cenário onde a busca pelo aumento de produtividade e desempenho operacional dos fornos elétricos é constante, os painéis duplos foram desenvolvidos para permitir maior continuidade / estabilidade operacional dos fornos, haja visto que paradas de produção de caráter corretivo para substituição de painéis refrigerados do forno em função de vazamentos de água, de modo geral, duram no mínimo 120 minutos.

Dentro de uma mesma corrida cada etapa do processo possui um perfil térmico característico, variando em grande escala o aporte térmico (fluxo de calor) de exposição dos painéis refrigerados. Ou seja, o aporte térmico ao qual o painel é submetido na etapa de fusão da carga sólida não é o mesmo que o painel está exposto na etapa de refino, quando há a cobertura do banho pela escória espumante. A esta variação de temperatura a qual os painéis refrigerados são expostos ao longo do processo caracteriza o ciclo térmico do FEA, que possui grande influência na ocorrência de fadiga térmica.

A fadiga nos tubos dos painéis refrigerados do FEA é um fenômeno que ocorre em função do aquecimento desigual de partes deste componente. As tensões térmicas as quais os painéis refrigerados são acometidos podem levar estes equipamentos à falha por fadiga, que neste caso é denominada fadiga térmica [2].

Outro mecanismo de falhas em painéis refrigerados é através de curto circuito elétrico, em função do sistema elétrico do FEA. O comportamento de um arco elétrico em um sistema trifásico é tido como caótico, pois envolve uma rápida e irregular mudança na geometria do arco devido à convecção, aos jatos de plasma e às forças eletromagnéticas. A priori os painéis refrigerados estão posicionados a uma distância "operacionalmente segura" dos eletrodos do FEA e em grande parte dos FEA os painéis refrigerados estão conectados ao circuito de aterramento do forno, ficando teoricamente protegidos dos efeitos causados pelo arco elétrico dos eletros do FEA. Porém, outros fatores como o uso de sucatas de grande comprimento e alta densidade, a má distribuição da carga de sucata no interior do FEA e parâmetros elétricos desregulados podem contribuir para a exposição dos painéis refrigerados aos danos por arco elétrico nos FEA [1].

O rebote de chamas e/ou splash de oxigênio é caracterizado por uma mudança brusca de direção e sentido dos gases injetados no forno através dos equipamentos de energia química, como injetores e queimadores. Neste mecanismo de falha os 
danos provocam a redução da espessura da parede do tubo do painel refrigerado e consequentemente altera a taxa de transferência de calor por condutividade na parede do tubo, o que deveria provocar o aumento da temperatura da água de refrigeração. Porém, a falha do painel refrigerado (vazamento de água no painel) geralmente ocorre antes que seja possível detectar o aumento de temperatura da água do painel [2].

\section{MATERIAIS E MÉTODOS}

A metodologia utilizada do desenvolvimento deste trabalho foi baseada na análise estatística de dados históricos das intervenções de manutenção nos painéis refrigerados do FEA ao longo do ano de 2014.

Uma vez conhecidas as principais causas de intervenções em painéis refrigerados do FEA e em qual(is) posição(ões) estas intervenções ocorriam com maior frequência o planejamento do experimento foi realizado, objetivando-se a redução dos números de intervenções, aumento da confiabilidade e da segurança operacional, aumento de disponibilidade dos equipamentos e redução de custos.

\subsection{Definição da Posição do FEA}

A análise dos dados e históricos de manutenção referentes ao ano de 2014 apontaram a Posição 10 do FEA como sendo a posição mais crítica para montagem de painéis refrigerados. Esta comprovação pode ser observada através do gráfico de Pareto apresentado na Figura 1.

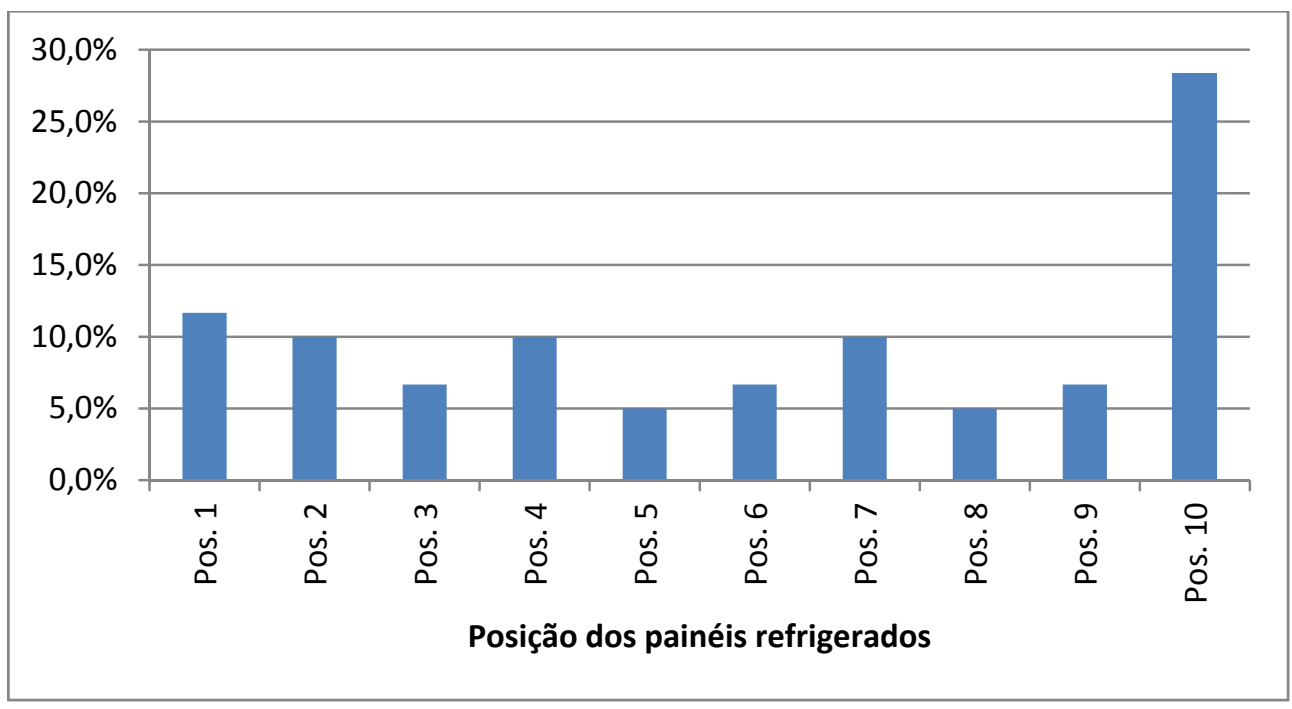

Figura 1. Percentual de ocorrências e intervenções nos painéis refrigerados do FEA ao longo do ano de 2014, por posição de montagem.

O gráfico apresentado na Figura 2 apresenta, em termos percentuais, as principais causas de ocorrências no painel da Posição 10. Nota-se que apenas $6 \%$ das trocas de painéis nesta posição foram realizadas de forma preventiva, em função do fim da vida útil estipulada para o mesmo. 


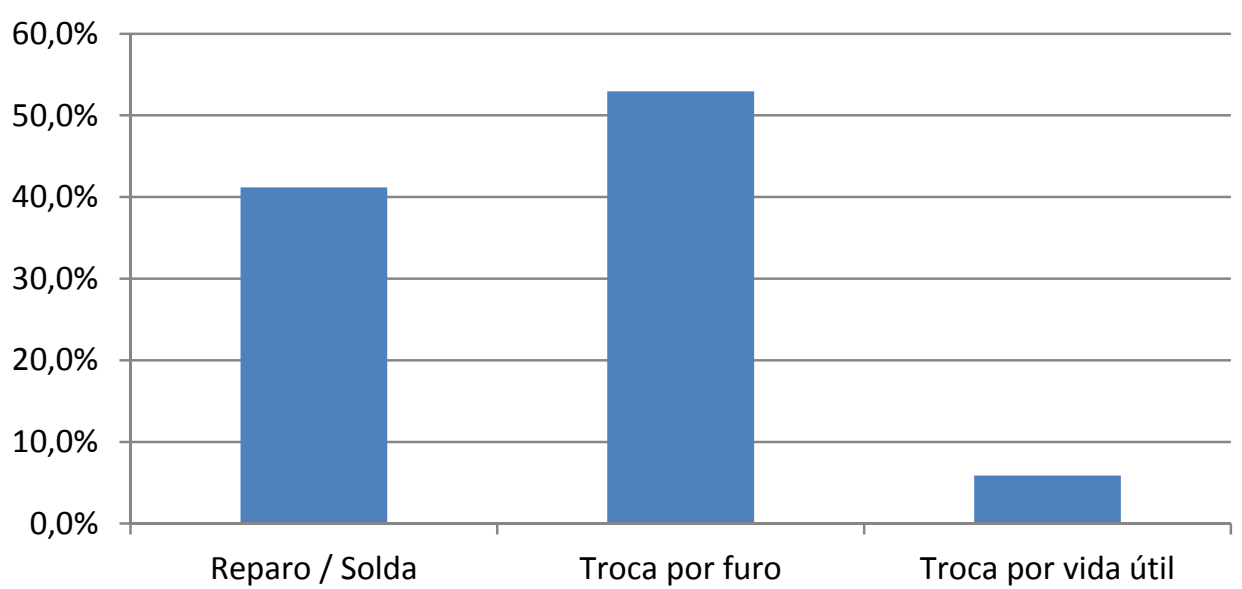

Tipo de ocorrência no painel refrigerado

Figura 2. Percentual dos tipos de ocorrências e intervenções no painel refrigerado da Pos.10 do FEA ao longo do ano de 2014.

O painel refrigerado que foi utilizado na Posição 10 ao longo do ano de 2014 era um duplo fabricado com tubos de aço carbono. Porém, em função das características construtivas, mesmo sendo um painel duplo, o modelo de painel utilizado não permite que o circuito frontal seja "sacrificado" (água de refrigeração desligada) e que o FEA continue operando com este painel tendo apenas com um circuito de refrigeração traseiro. Por isso, as ocorrências de vazamentos no painel sempre requerem a substituição imediata dos mesmos, o que demanda maior tempo de interrupção.

Por estes motivos que a Posição 10 do FEA foi escolhida para receber a primeira instalação dos painéis refrigerados da tecnologia Double Side, da empresa Lumar Metals, na Votorantim Resende.

\subsection{Gerenciamento da Mudança}

Como seria testado um novo conceito de painel refrigerado, foi necessária a elaboração de um "Formulário de Gerenciamento de Mudança", visando identificar quais impactos esta mudança poderia causar na segurança operacional e na forma de operar dos colaboradores. A Votorantim Siderurgia possui um formulário específico para este propósito, conforme apresentado na Figura 3.

Através da utilização desta ferramenta, o principal ponto observado foi que havia uma mudança considerável na configuração e montagem das mangueiras de refrigeração dos circuitos do painel, bloco e injetor. Foi observado que estas alterações poderiam causar falhas graves durante a montagem e manutenção do conjunto, bem como falha durante o procedimento de sacrifício do painel, caso este procedimento fosse necessário. Qualquer que fosse o tipo da falha, esta poderia causar consequências fortes, como uma explosão no FEA. Desta forma, foi elaborado um material técnico com fotos, especificações, instruções e o passo-apasso da montagem dos equipamentos. O procedimento de sacrifício do painel também foi contemplado no material elaborado. Todos os colaboradores do FEA e do setor de manutenção foram treinados in loco. 


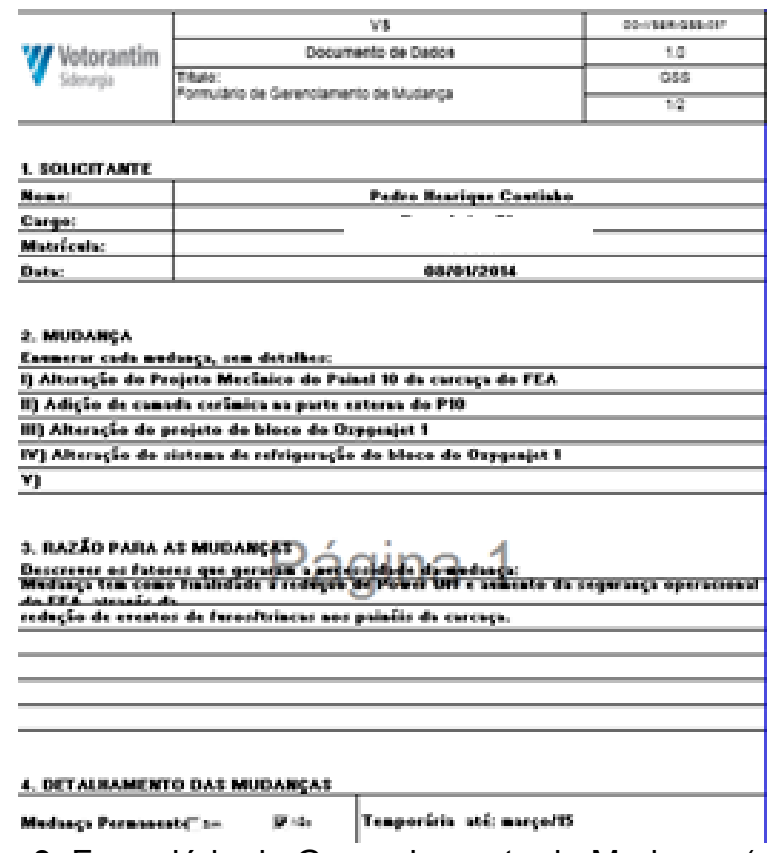

Figura 3. Formulário de Gerenciamento de Mudança (parcial)

Além do treinamento operacional da equipe de colaboradores da Votorantim, também foi realizado o start up e operação assistida dos equipamentos pelas equipes técnicas da Votorantim e Lumar Metals nos primeiros dias de operação.

\subsection{Planejamento do Experimento}

\begin{tabular}{|c|c|c|c|}
\hline \multirow{4}{*}{ VV Votorantim } & \multirow{2}{*}{$\begin{array}{l}\text { VOTORANTIM SIDERURGIA } \\
\text { DOCUMENTO DE DADOS }\end{array}$} & Código & $\begin{array}{c}\text { DD-VSRS-GAE- } \\
001\end{array}$ \\
\hline & & Número & $001 / 2012$ \\
\hline & \multirow{2}{*}{ PLANEJAMENTO DE EXPERIMENTO } & Área & GAE \\
\hline & & Páginas & $1 / 6$ \\
\hline
\end{tabular}

Serão modificados o Painel 10,Figura 1, que terá um acréscimo de uma camada cerámica em sua face frontal (Double Side), e o bloco de cobre do OJ1, que teráa área de exposiçảo reduzida e maior proteçăo
contra rebotes por oxigênio (Protect Block), conforme apresentado na Figura 2 .

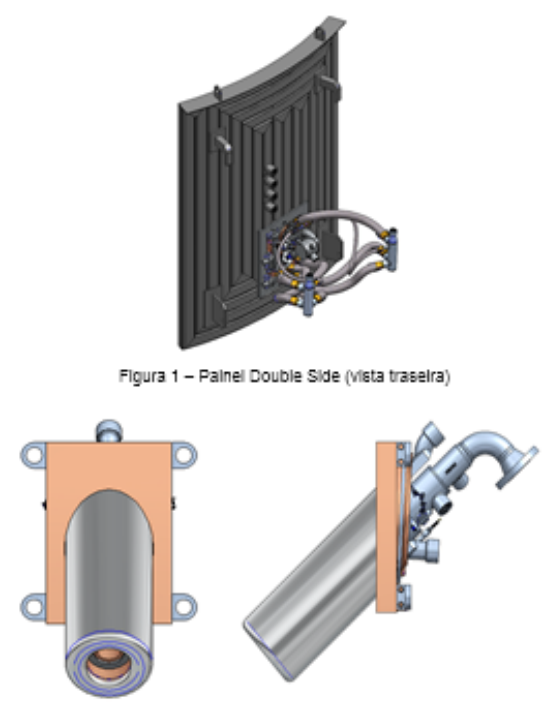

Flgura 2 - Protect Block (Msta frontal e lateral).

Figura 4. Planejamento de experimento (reprodução parcial). 
Para a realização do teste também foi elaborado o "Planejamento de Experimento". Esta ferramenta tem como objetivo definir as condições em que o teste será realizado, a duração do período de teste, o cronograma de atividades, quais as variáveis a serem monitoradas, quais as variáveis críticas do teste, quais parâmetros de avaliação do teste, quais os principais pontos de atenção, quem são os responsáveis pelo teste, o que fazer em caso de emergência. Tudo isto foi importante para garantir que o teste tivesse sucesso e pudesse ser representativo para aprovar ou não o material que seria testado. Parte do Planejamento de Experimento pode ser visto na Figura 4.

\subsection{Painel Double Side e Protect Block}

Double Side é uma nova tecnologia de painéis refrigerados desenvolvida pela Lumar Metals especialmente para aplicações em condições severas de operação do FEA, tais como pontos quentes químicos e elétricos. O desenho do painel pode ser visto na Figura 5. As principais características dos painéis refrigerados da tecnologia Double Side são:

- Circuito de refrigeração customizado e otimizado;

- Os painéis Double Side são painéis duplos, com circuito frontal e traseiro totalmente independentes;

- Dimensões reduzidas. A espessura dos painéis Double Side é inferior aos painéis duplos convencionais, o que permite maior aproveitamento do volume interno do FEA;

- Revestimento face frontal do painel, permitindo maior resistência às intempéries do FEA (rebotes de chama, curto-circuito elétrico, impactos, altas temperaturas) e conferindo vida longa ao painel, quando comparado aos painéis tubulares convencionais.

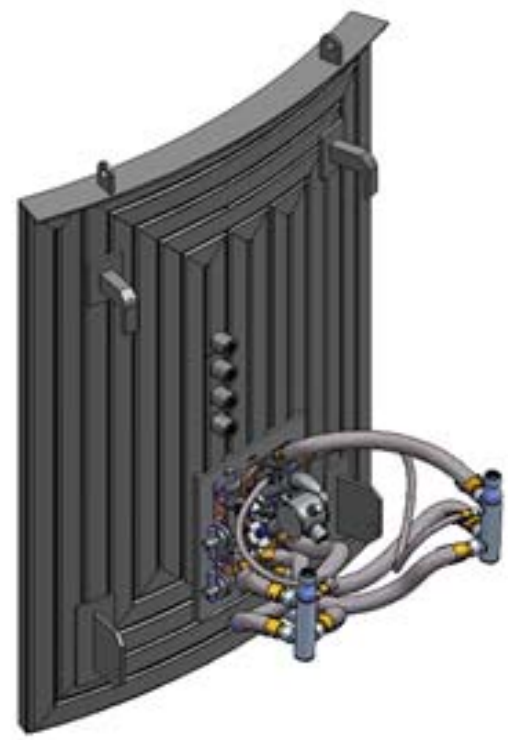

Figura 5. Vista traseira do painel Double Side.

O Protect Block, de tecnologia Lumar Metals, é um bloco de cobre refrigerado de design compacto, porém capaz de aproximar os injetores do banho de aço líquido, promovendo ganhos metalúrgicos ao processo, Figura 6. A menor área de exposição do bloco aliada ao sistema de refrigeração otimizado reduz as possibilidades de danos ao bloco durante operação. 

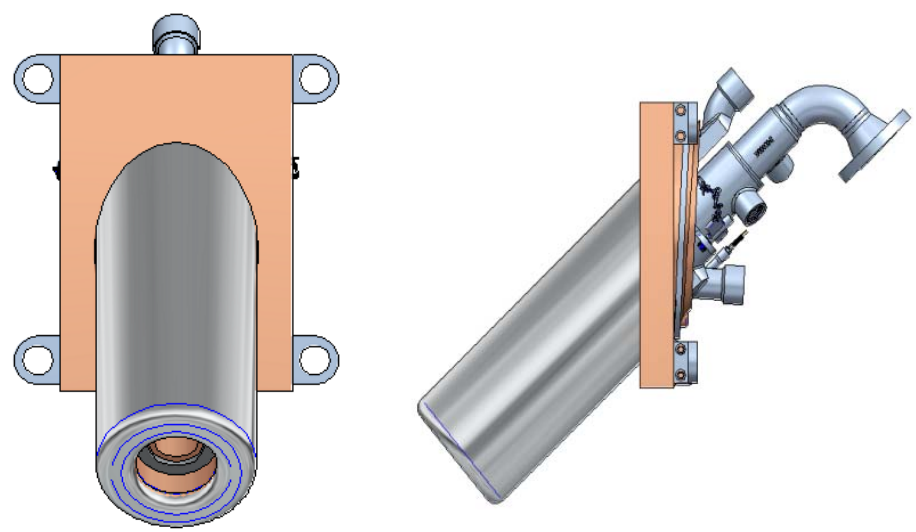

Figura 6. Imagem esquemática do do Protect Block - Vista frontal e lateral.

As principais características do Protect Block são:

- Redução de peso de aproximadamente $50 \%$, quando comparado aos blocos de cobre convencionais;

- Menor área de exposição no interior do FEA;

- Camisa refrigerada capaz de resistir a impactos diretos de até $3.000 \mathrm{~kg}$;

- Sistema de refrigeração otimizado para garantir rápida troca térmica;

- Sistema de montagem modular, composto de suporte frame, frame e camisa refrigerada.

\section{RESULTADOS E DISCUSSÃO}

Durante a evolução dos testes a principal variável monitorada foi a temperatura de saída da água de refrigeração dos circuitos do painel da Posição 10, principalmente a temperatura de saída da água de refrigeração do circuito frontal do painel, totalmente exposto às condições agressivas do FEA.

O gráfico da Figura 7 apresenta um comparativo da evolução da temperatura da água de saída do painel ao longo de uma corrida típica do FEA, no modelo de painel tubular convencional e do painel Double Side, ambos na posição 10 do FEA.

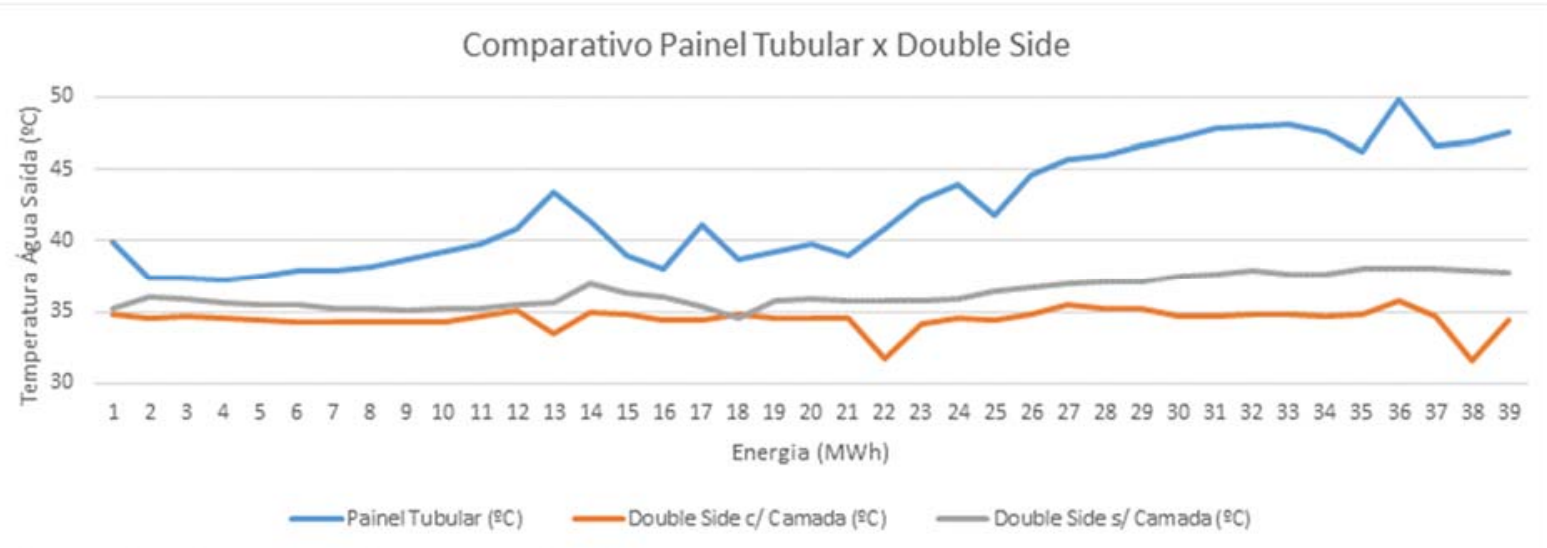

Figura 7. Perfil de Temperatura na posição 10 com o Painel Tubular (azul), Double Side com camada cerâmica (laranja) e Double Side sem Camada Cerâmica (cinza).

Observa-se pelo gráfico que o painel tubular convencional tem uma elevação da temperatura da água no período final de fusão da primeira carga do FEA e logo após, durante fusão do segundo carregamento até o final do refino da corrida, há a 
elevação progressiva da temperatura da água de refrigeração do painel. A temperatura do painel Double Side foi monitorada em duas condições distintas de operação, com e sem revestimento cerâmico. Em ambas situações a temperatura de água do painel Double Side mínima variação ao longo da corrida.

O resultado obtido com o painel Double Side já era esperado, pois estudos preliminares realizados através de simulação computacional mostraram que, para uma mesma condição operacional (vazão de água, aporte térmico, etc.), quando comparado aos painéis convencionais, a temperatura da água de saída do painel Double Side é mais de quatro vezes menor do que em um painel convencional, conforme mostrado na Figura 8.

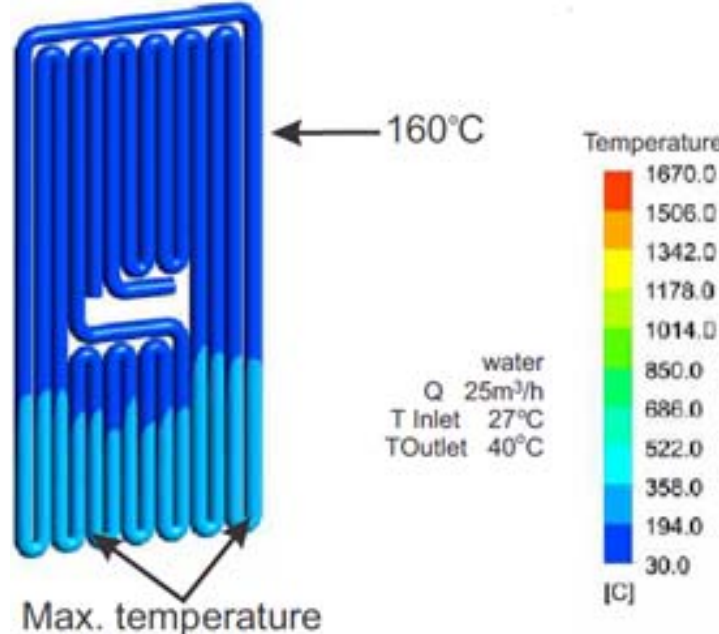

$319^{\circ} \mathrm{C}$ exposed

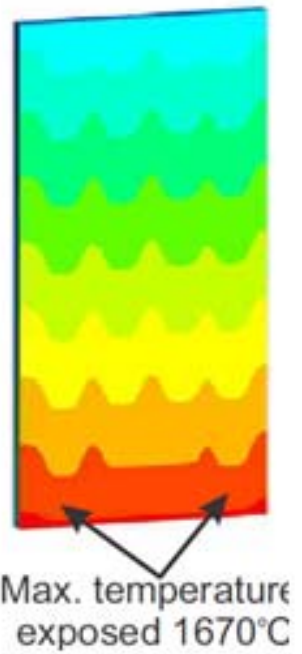

exposed $1670^{\circ} \mathrm{C}$

Figura 8. Resultado de simulação computacional comparando um painel tubular convencional (figura da esquerda) com um painel da tecnologia Double Side (figura da direita), ambos com as mesmas condições de operação.

Como a temperatura da água de saída do Painel Double Side é menor, pode-se afirmar também que ele retira menos calor do processo do FEA. Fazendo uma simulação em uma situação típica, a quantidade de calor extraída pelo Double Side seria de $87 \mathrm{~kJ} / \mathrm{s}$, enquanto o painel tubular teria uma extração de $376 \mathrm{~kJ} / \mathrm{s}$. Esta é uma observação importante, pois pode contribuir para a redução do consumo de energia do FEA e também para redução de formação de zonas frias no FEA (regiões de sucata não fundida).

Baseado no perfil térmico apresentado pelo painel Double Side, como a variação de temperatura é menor (quando comparado ao tubular), menor é a ocorrência de contração / dilatação no painel. Dessa forma, as ocorrências de trincas por fadiga térmica são minimizadas. Na Tabela 1 , segue comparação da média, desvio padrão e pico de temperatura da água de saída para o painel tubular convencional e o Double Side.

Tabela 1. Parâmetros operacionais dos painéis na posição 10 do FEA.

\begin{tabular}{lccc}
\hline & Temp. Média & Temp. Máxima & Desvio padrão \\
\hline Painel Tubular & $41,8^{\circ} \mathrm{C}$ & $59^{\circ} \mathrm{C}$ & 4,98 \\
\hline Double Side c/ Camada & $34,5^{\circ} \mathrm{C}$ & $37^{\circ} \mathrm{C}$ & 1,74 \\
\hline Double Side s/ Camada & $36,3^{\circ} \mathrm{C}$ & $41^{\circ} \mathrm{C}$ & 2,00 \\
\hline
\end{tabular}


O Protect Block é vantajoso operacionalmente, pois sua área de exposição é menor, diminuindo a probabilidade de rebotes, além disso foi possível trocar a camisa refrigerada (similar ao bloco de cobre) sem trocar o painel refrigerado. Este resultado é muito importante, pois atualmente caso haja algum evento no bloco de cobre, é necessário realizar a troca de painel e que dura entre 90 a 120 minutos, enquanto foi possível trocar a camisa refrigerada com apenas 30 minutos.

Em resumo, a utilização do Protect Block é importante para a redução do número de eventos de furo de bloco, além da redução do MTTR (Mean Time to Repair) em relação à troca do bloco/camisa refrigerada. A utilização deste modelo também contribui para aumento da campanha do painel de aço, pois como explicado no parágrafo acima, em alguns momentos o painel é retirado de maneira prematura também devido aos eventos no bloco de cobre.

Segue abaixo evolução da vida dos painéis da posição 10, na Figura 9. Conforme gráfico, houve aumento da vida do painel da posição 10 , cerca de $30 \%$ acima da meta. A últimas 4 campanhas sequer haviam atingido a meta de troca do painel.

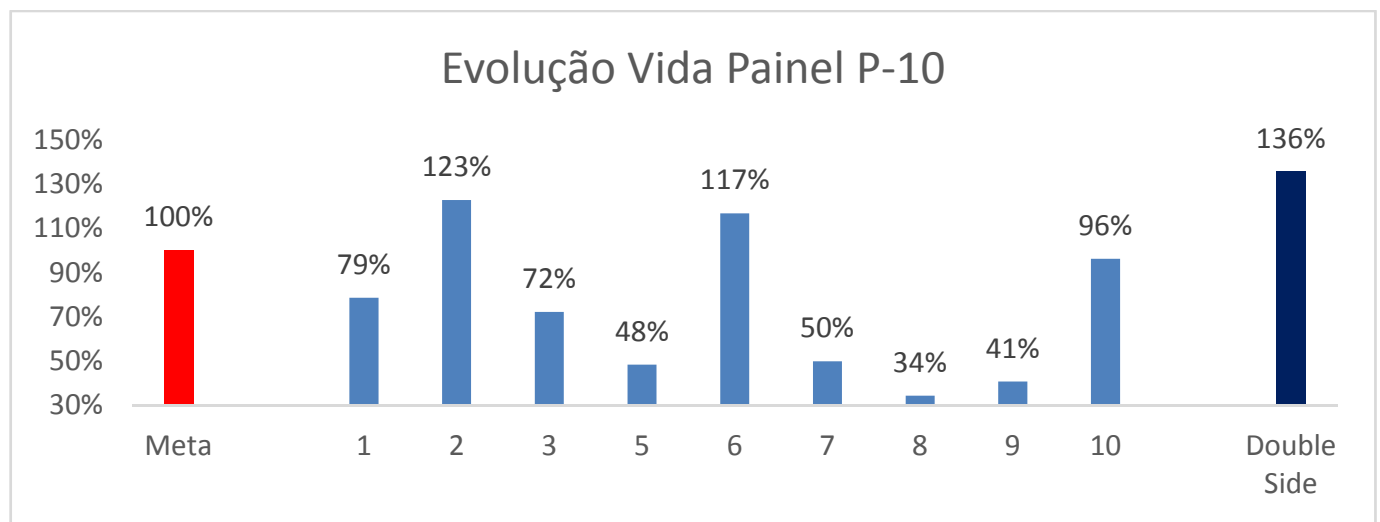

Figura 9. Evolução da vida dos painéis da posição 10.

\section{CONCLUSÃO}

A partir do teste realizado com as tecnologias Double Side e Protect Block foi possível chegar as seguintes conclusões:

- Ambas tecnologias conseguiram suportar o ambiente agressivo de um FEA com potência elétrica e aporte químico elevados.

- O painel Double Side apresentou um perfil térmico superior ao painel tubular, minimizando a probabilidade de ocorrência de trinca térmica, até mesmo sem o revestimento cerâmico.

- O painel Double Side extrai menos calor do FEA do que um painel tubular.

- No FEA da Votorantim Resende, foi possível trocar o bloco de cobre (no caso, camisa refrigerada) sem necessidade de troca do painel de aço.

- No primeiro teste, a vida do painel superou a meta em $36 \%$.

A próxima etapa deste trabalho, previsto pelo Planejamento do Experimento, é a realização da perícia do painel Double Side e do Protect Block que saíram de operação do FEA após o teste piloto. Após análise da perícia e avaliação técnica dos equipamentos os testes continuarão em outra posição do FEA. 


\section{REFERÊNCIAS}

1 Silveira, R. C. da, Fabricação de Aços em Fornos Elétricos. Editora UFOP. Minas Gerais, Brasil, 1997.

2 Wandekoken, T. P., Maia, B. T. Fundamentos Básicos da Operação e Manutenção de Painéis Refrigerados de Fornos Elétricos a Arco - FEA. Lumar - Treinamento Interno. Minas Gerais, Brasil, 2013. 